\title{
The generalized second law of thermodynamics in generalized gravity theories
}

\author{
Shao-Feng $\mathrm{Wu}^{1 *}$, Bin $\mathrm{Wang}^{2 \dagger}$, Guo-Hong Yang ${ }^{1 \ddagger}$, and Peng-Ming Zhang, 3 § \\ ${ }^{1}$ Department of Physics, Shanghai University, Shanghai, 200436, P. R. China \\ ${ }^{2}$ Department of Physics, Fudan University, Shanghai 200433, P. R. China \\ ${ }^{3}$ Center of Theoretical Nuclear Physics, \\ National Laboratory of Heavy Ion Accelerator, Lanzhou 730000, P. R. China and \\ ${ }^{4}$ Institute of Modern Physics, Lanzhou, 730000, P. R. China
}

\begin{abstract}
We investigate the generalized second law of thermodynamics (GSL) in generalized theories of gravity. We examine the total entropy evolution with time including the horizon entropy, the non-equilibrium entropy production, and the entropy of all matter, field and energy components. We derive a universal condition to protect the generalized second law and study its validity in different gravity theories. In Einstein gravity, (even in the phantom-dominated universe with a Schwarzschild black hole), Lovelock gravity, and braneworld gravity, we show that the condition to keep the GSL can always be satisfied. In $f(R)$ gravity and scalar-tensor gravity, the condition to protect the GSL can also hold because the gravity is always attractive and the effective Newton constant should be approximate constant satisfying the experimental bounds.

PACS numbers: 04.70.Dy, 04.50.-h, 98.80.-k
\end{abstract}

\footnotetext{
* Corresponding author. Email: sfwu@shu.edu.cn; Phone: +86-021-66136202.

† Email: wangb@fudan.edu.cn

¥ Email: ghyang@mail.shu.edu.cn

§ Email: zhpm@impcas.ac.cn
} 


\section{INTRODUCTION}

Motivated by the black hole physics, it was realized that there is a profound connection between gravity and thermodynamics. In Einstein gravity, the evidence of this connection was first discovered in [1] by deriving the Einstein equation from the proportionality of entropy and horizon area together with the first law of thermodynamics in the Rindler spacetime. For a general static spherically symmetric spacetime, Padmanabhan pointed out that Einstein equations at the horizon give rise to the first law of thermodynamics [2]. Recently the study on the connection between gravity and thermodynamics has been extended to cosmological context. Frolov and Kofman [3] employed the approach proposed by Jacobson [1] to a quasi-de Sitter geometry of inflationary universe, and calculated the energy flux of a background slow-roll scalar through the quasi-de Sitter apparent horizon. By applying the first law of thermodynamics to a cosmological horizon, Danielsson obtained Friedmann equation in the expanding universe [4]. In the quintessence dominated accelerating universe, Bousso [5] showed that the first law of thermodynamics holds at the apparent horizon. The relation between gravity and thermodynamics has been further disclosed in extended gravity theories, including Lovelock gravity [6, 7], braneworld gravity [8, 9], nonlinear gravity [7, 10, 11], and scalar-tensor gravity [7, 11] etc. In the nonlinear gravity and scalar-tensor gravity, it was argued that the non-equilibrium thermodynamics instead of the equilibrium thermodynamics should be taken into account to build the relation to gravity [7, 10, 11]. In our previous work [12], we have presented a general procedure to build the connection between gravity and thermodynamics. From the Friedmann equations, we have constructed the first law of thermodynamics on the apparent horizon in generalized gravity theories. We found that the non-equilibrium entropy production term arising in non-linear gravity and scalar-tensor gravity is due to the existence of other dynamic fields besides the ordinary matter dominating the cosmological evolution.

It is of great interest to extend our discussion in [12] to study the generalized second law (GSL) of thermodynamics in the generalized gravity theories. There have been a lot

of interest on investigating the GSL in gravity [13, 14, 15, 16, 17, 18, 19, 20, 21], but all of them concentrate on the Einstein gravity. The modified theory of gravity was argued to be a possible candidate to explain the accelerated expansion of our universe, thus it is interesting to examine the GSL in the extended gravity theories. An attempt to study 
this problem was carried out in [22], where it was found that some additional conditions are needed for validity of GSL. Even for the Einstein gravity, it was found that GSL breaks down in phantom-dominated universe in the presence of Schwarzschild black hole [18], at least in transition epoch [19]. In our paper we will adopt the formalism proposed in [12]. We will derive the entropy of the horizon from the first law of thermodynamics constructed in [12]. We will examine the total entropy evolution with time including the horizon entropy, the nonequilibrium entropy production, and the entropy of all matter, field and energy components. We will derive a universal condition to protect the GSL in generalized gravity theories and examine its validity in the Einstein gravity (even in the presence of Schwarzschild black hole), Lovelock gravity, braneworld gravity, nonlinear gravity and scalar-tensor gravity.

The organization of the paper is as follows: In section 2, we briefly review the generalized first law of thermodynamics in extended theories of gravity. In section 3, we derive the universal condition to protect the GSL and examine its validity in some extended gravity theories. The last section is devoted to summary.

\section{THE FIRST LAW OF THERMODYNAMICS ON THE APPARENT HORIZON IN FRW COSMOLOGY}

In this section we briefly go over the general procedure to construct the first law of thermodynamics on the apparent horizon in generalized gravity theory [12].

The homogenous and isotropic $(n+1)$-dimensional FRW universe is described by

$$
d s^{2}=h_{a b} d x^{a} d x^{b}+\tilde{r}^{2} d \Omega_{n-1}^{2}
$$

where $h_{a b}=\operatorname{diag}\left(-1, \frac{a^{2}}{1-k a^{2}}\right), d \Omega_{n-1}^{2}$ is the $(n-1)$-dimensional sphere element, and $x^{0}=$ $t, x^{1}=r, \tilde{r}=a r$ is the radius of the sphere and $a$ is the scale factor. For simplicity, we consider the flat space $k=0$ in this paper, however, our discussion can also be generalized to the non-flat cases. It is known that the dynamical apparent horizon, the marginally trapped surface with vanishing expansion, is defined as a sphere situated at $r=r_{A}$ satisfying

$$
h^{a b} \partial_{a} \tilde{r} \partial_{b} \tilde{r}=0 .
$$

The sphere radius is

$$
\tilde{r}_{A} \equiv r_{A} a=\frac{1}{H} .
$$


The associated temperature on the apparent horizon is defined by surface gravity $\kappa=$ $\frac{1}{\sqrt{-h}} \partial_{a}\left(\sqrt{-h} h^{a b} \partial_{b} \tilde{r}\right)$

$$
T=\frac{|\kappa|}{2 \pi}=\frac{1}{2 \pi \tilde{r}_{A}}(1-\epsilon)
$$

where $\epsilon \equiv \frac{\partial_{t} \tilde{r}_{A}}{2 H \tilde{r}_{A}}<1 . \epsilon<1$ ensures that the temperature is positive. Using the definition of the horizon (2), the positive temperature condition can be written as $\epsilon=-\frac{\dot{H}}{2 H^{2}}<1$, i.e.

$$
\dot{H}>-2 H^{2},
$$

which is useful in our later discussion. In our previous work [12], in order to study the mass-like function, we construct the first law on the assumption $\epsilon \ll 1$ meaning that the apparent horizon radius is approximately fixed thereby the temperature $T=\frac{1}{2 \pi \tilde{r}_{A}}[7,23]$. Here we will drop this assumption.

In Einstein gravity, the entropy is proportional to the horizon area

$$
S_{E}=\frac{A}{4 G},
$$

where the horizon area $A=n \Omega_{n} \tilde{r}_{A}^{n-1}$. The thermodynamical fluid $\delta Q$ can be written as

$$
T d S_{E}=\frac{n(n-1) V \tilde{r}_{A}^{-3} d \tilde{r}_{A}}{8 \pi G}-\frac{n(n-1) V \tilde{r}_{A}^{-3} d \tilde{r}_{A}}{8 \pi G} \frac{\partial_{t} \tilde{r}_{A}}{2 H \tilde{r}_{A}},
$$

where $V=\Omega_{n} \tilde{r}_{A}^{n}$ is the volume in the horizon. Using the definition of the horizon (2) and the temperature (3), we can obtain

$$
T d S_{E}=\frac{-n(n-1) V}{16 \pi G} \frac{d H^{2}}{d t} d t-\frac{n(n-1) V}{16 \pi G} \frac{\dot{H}^{2}}{H} d t,
$$

which is purely a geometric relation.

In all gravity theories, Friedmann equations can be expressed in the form as that in the Einstein gravity

$$
\begin{gathered}
H^{2}=\frac{16 \pi G}{n(n-1)} \rho_{e f f} \\
\dot{H}=-\frac{8 \pi G}{(n-1)}\left(\rho_{e f f}+p_{e f f}\right) .
\end{gathered}
$$

Though we do not know the exact form of $\rho_{\text {eff }}$ (and $p_{\text {eff }}$ ), we know that there must be ordinary matter density $\rho$ in $\rho_{\text {eff }}$ and also other variables $\rho_{p}$. In some cases, $\rho_{p}$ (or their combination) may describe other matter field $\rho_{f}$ or effective energy component $\rho_{e}$ besides the ordinary matter $\rho$ in $\rho_{\text {eff }}$. The first Friedmann equation can be expressed in the form

$$
H^{2}=H^{2}\left(\rho, \rho_{1}, \cdots \rho_{p}, \cdots\right)
$$


Then the relation (5) can be changed as

$$
T d S_{E}=\frac{-n(n-1) V}{16 \pi G} d t\left(\frac{\partial H^{2}}{\partial \rho} \dot{\rho}+\frac{\partial H^{2}}{\partial \rho_{p}} \dot{\rho}_{p}\right)-\frac{n(n-1) V}{16 \pi G} \frac{\dot{H}^{2}}{H} d t .
$$

To construct the first law of thermodynamics, we need to know the energy flux $d E$ or entropy $S$. In the general gravity theory, they are not specified. However, it is known that the energy flux of ordinary matter includes $V \dot{\rho} d t$. Multiplying $\frac{16 \pi G}{n(n-1)} \frac{1}{\frac{\partial H^{2}}{\partial \rho}}$ on both sides of (8) , we can extract it clearly

$$
\frac{16 \pi G}{n(n-1)} \frac{1}{\frac{\partial H^{2}}{\partial \rho}} T d S_{E}=-V \dot{\rho} d t-V d t \frac{1}{\frac{\partial H^{2}}{\partial \rho}} \frac{\dot{H}^{2}}{H}-V d t \frac{1}{\frac{\partial H^{2}}{\partial \rho}} \frac{\partial H^{2}}{\partial \rho_{p}} \dot{\rho}_{p} .
$$

In the general case, we have the conservation

$$
\dot{\rho}_{e f f}+n H\left(\rho_{e f f}+p_{e f f}\right)=0 .
$$

We furthermore assume that ordinary matter (All matter and energy in this paper are assumed as perfect fluid) has energy exchange with other energy source, described by

$$
\dot{\rho}+n H(\rho+p)=q,
$$

If the gravity theory has matter $\rho_{f}$ and energy content $\rho_{e}$, one may also have similar semiconserved laws

$$
\begin{gathered}
\dot{\rho}_{f}+n H\left(\rho_{f}+p_{f}\right)=q_{f}, \\
\dot{\rho}_{e}+n H\left(\rho_{e}+p_{e}\right)=q_{e}, \\
\dot{\rho}_{t}+n H\left(\rho_{t}+p_{t}\right)=q_{t} .
\end{gathered}
$$

In the last equation the total density $\rho_{t} \equiv \rho+\rho_{f}+\rho_{e}$, total pressure $p_{t} \equiv p+p_{f}+p_{e}$, and total energy exchange $q_{t} \equiv q+q_{f}+q_{e}$ are introduced. However, it should be emphasized that one can not impose total energy fluid $q_{t}=0$, because there may be energy exchange with the horizon. Equations (10) and (11) will be used later to express the first law explicitly. Since we will consider thermodynamical effect with the change of horizon volume, we introduce the work density. Defining $T_{a}^{b}$ as the projection of energy-momentum tensor $T_{\nu}^{\mu}$ of the perfect fluid in the FRW universe in the normal direction of the $(n-1)$-sphere, we have the density $W \equiv-\frac{1}{2} T_{a}^{a}$, which may be viewed as the work done by the change of the apparent horizon, as pointed out in [36]. 
Consider the entropy change should be an exact form in the first law of thermodynamics. If there is just ordinary matter $\rho$ in the space, $\frac{\partial H^{2}}{\partial \rho}$ can be rewritten as a function of $\tilde{r}_{A}$. Then a total differential can be obtained by the integration

$$
S=\int \frac{16 \pi G}{n(n-1)} \frac{1}{\frac{\partial H^{2}}{\partial \rho}\left(\tilde{r}_{A}\right)} d\left(S_{E}\right)
$$

and the relation (9) can be written as

$$
T d S=\delta Q
$$

where the thermodynamical fluid

$$
\delta Q=-V \dot{\rho} d t-V d t \frac{1}{\frac{\partial H^{2}}{\partial \rho}} \frac{\dot{H}^{2}}{H} .
$$

Since the gravity is only determined by ordinary matter, we can impose conservation $q=0$. Using the conservation equation (11), one can prove

$$
-V \frac{1}{\frac{\partial H^{2}}{\partial \rho}} \frac{\dot{H}^{2}}{H}=-\frac{1}{2}(\rho+p) \dot{V} .
$$

Then the thermodynamical fluid can be rewritten as

$$
\delta Q=-d E+W d V
$$

which includes just the energy flux of ordinary matter $E=\rho V$ and the work done by the change of the apparent horizon. Thus the expression (13) should be understood as the entropy to assure the first law

$$
T d S=-d E+W d V
$$

If the modified gravity theory has other dynamic fields resulting that $\frac{\partial H^{2}}{\partial \rho}$ is a function of $\tilde{r}_{A}$ and $\rho_{p}$

$$
\frac{\partial H^{2}}{\partial \rho}=\frac{\partial H^{2}}{\partial \rho}\left(\tilde{r}_{A}, \rho_{p}\right)
$$

we can not integral the l.h.s in (9) directly. However we can express it as

$$
T \frac{16 \pi G}{n(n-1)} \frac{1}{\frac{\partial H^{2}}{\partial \rho}} d S_{E}=T d\left(\frac{16 \pi G}{n(n-1)} \frac{1}{\frac{\partial H^{2}}{\partial \rho}} S_{E}\right)-T \frac{16 \pi G}{n(n-1)} S_{E} d \frac{1}{\frac{\partial H^{2}}{\partial \rho}}
$$

It can be rewritten as

$$
T d S=\delta Q
$$


where

$$
\begin{gathered}
S \equiv \frac{16 \pi G}{n(n-1)} \frac{1}{\frac{\partial H^{2}}{\partial \rho}} S_{E}, \\
\delta Q \equiv-V \dot{\rho} d t-V d t \frac{1}{\frac{\partial H^{2}}{\partial \rho}} \frac{\dot{H}^{2}}{H}-V d t \frac{1}{\frac{\partial H^{2}}{\partial \rho}} \frac{\partial H^{2}}{\partial \rho_{p}} \dot{\rho}_{p}+T \frac{16 \pi G}{n(n-1)} S_{E} d \frac{1}{\frac{\partial H^{2}}{\partial \rho}} .
\end{gathered}
$$

To construct the first law, we can write

$$
\delta Q=-d E+W_{t} d V-T d_{p} S
$$

where $E \equiv \rho_{t} V$ is the total intrinsic energy and $W_{t}$ the total work. $d_{p} S$ is defined as

$$
d_{p} S \equiv-\frac{1}{T}\left(\delta Q+d E-W_{t} d V\right)
$$

where the subindex "p" denotes the term resulted from other dynamical fields. In general, $d_{p} S$ is non-vanishing and can not be written out exactly (We will show $d_{p} S$ clearly in concrete gravity theories). If the first law of generalized gravity theory can be constructed, the entropy term contains the form (19) together with the entropy production $d_{p} S$ (21) following the idea given in [10]. Here we do not consider whether the entropy is developed internally by the system as in [10]. The first law is expressed as

$$
T d S+T d_{p} S=-d E+W_{t} d V
$$

The exact forms of entropy production $d_{p} S$ and entropy $S$ depend on the concrete gravity theory.

In [27] it was argued that the entropy correction can be absorbed in the mass-like function and the entropy generation in the nonequilibrium can be reinterpreted. Whether one should interpret the thermodynamical equations as representing systems in equilibrium or out of equilibrium seems unclear. This is also under debate in recent works on $f(R)$ gravity [28, 29]. In our case, there is work term, since the horizon is not fixed as that in [27] and the form of the mass-like function is not known. The entropy expression, inner energy and the work term in the first law Eq. (22) are all determined. The extra term can not be absorbed into any other terms and it has to be interpreted as the entropy production as done in [10]. Since the mass-like function here is not available, we do not know how to reinterprete this entropy correction as done in [27].

One might argue that there seems ambiguities in entropy expressions (13) (19) since one might add a proper quantity to the expression of entropy, which may vanish in the 
Einstein gravity, and this extra term could be absorbed into the redefinition of the entropy production. This worry is not necessary. As pointed out in [12], the known black hole entropy in different gravity theories will strictly restrict the form of the additional quantities in the entropy expressions. The definition of entropy in (13) and (19) can recover the exact expression of the known black hole entropy [12] such as in Lovelock gravity [24], nonlinear

gravity [25] and scalar-tensor gravity [26]. Moreover, following the work in [27], the general mass-like function which can be reduced to the corresponding Misner-Sharp mass has been found. If we add other quantities in entropy expressions (13) (19), it seems very difficult to obtain the corresponding mass-like function which can be reduced to the known MisnerSharp mass. This serves as another restriction on adding additional terms to the entropy expressions.

\section{GSL OF EXTENDED GRAVITY THEORIES}

Now we start to discuss the GSL in generalized gravity theories. Most discussions on the GSL focus on the Einstein gravity. In generalized gravity theories, from the first law of thermodynamics (22) one can see that an entropy production term appears, which characterizes the non-equilibrium thermodynamical process about the horizon. In describing the GSL one should include this non-equilibrium entropy production term. Besides, in addition to the matter and fields, one should also include the entropy for the effective energy in describing the GSL, since the change of energy is relative to the change of entropy.

Now we are going to use the first law of thermodynamics (22) to find the general condition need to hold the GSL in any gravity theories. From (22), we have the expression of the horizon entropy

$$
T d S_{h}=-T d_{p} S-d E+W_{t} d V .
$$

On the other hand, if there is only ordinary matter inside the cosmological horizon of a comoving observer, the entropy of the ordinary matter is relative to the energy $\rho$ and the pressure $p$ via the Gibbs equation

$$
T_{\rho} d S_{\rho}=d(\rho V)+p d V=V d \rho+(\rho+p) d V .
$$

As pointed out in [30], the ordinary matter should be understood as a phenomenological representation of a mixture of fields, each of which may or may not be in a pure state, and 
therefore entitled to an entropy. $T_{\rho}$ refers to the temperature of matter inside the horizon. If there are other matter field and energy component, one can similarly have the Gibbs equation (including all matter, field, and energy contents)

$$
T_{t} d S_{t}=d\left(\rho_{t} V\right)+p_{t} d V=V d \rho_{t}+\left(\rho_{t}+p_{t}\right) d V
$$

Here the temperature of total energy inside the horizon is denoted as $T_{t}$. It should be noted that the temperature $T_{t}$ is not equal to $T$ in general because there may be energy flow $q_{t}$ (thereby thermodynamical fluid) between the horizon and energy inside the horizon. Since the only temperature scale we have at our disposal is the temperature of the apparent horizon $T$, we assume

$$
T_{t}=b T
$$

where the temperature parameter satisfying $0<b<1$ assures the temperature being positive and smaller than the horizon temperature. This ansatz is similar to the disposal when the horizon is taken as the event horizon [17, 30], where the only temperature scale is the de Sitter temperature [16].

Now, we propose that GSL should be expressed as

$$
\dot{S}_{h}+d_{p} \dot{S}+\dot{S}_{t} \geq 0
$$

where $d_{p} \dot{S} \equiv \partial_{t}\left(d_{p} S\right)$. Summing up Eqs. (23) and (25), the GSL reads

$$
-b \dot{E}+b W_{t} \dot{V}+V \dot{\rho}_{t}+\left(\rho_{t}+p_{t}\right) \dot{V}=(1-b) \dot{\rho}_{t} V+\left(1-\frac{b}{2}\right)\left(\rho_{t}+p_{t}\right) \dot{V} \geq 0 .
$$

It should be noted that if there is no energy flow $\left(q_{t}=0\right)$ between the horizon and energy therein, the horizon and the energy inside it are in thermal equilibrium $b=1$.

One can immediately find that the GSL holds for Einstein gravity. Two Friedmann equations (6) (17) make the energy flow $q$ in the continuity equation (11) vanish, thereby the thermal flow vanishes. Employing two Friedmann equations (마) (17) in condition (27) by replacing $\rho_{t}\left(p_{t}\right)$ as $\rho(p)$, we have

$$
-\dot{E}+W \dot{V}+V \dot{\rho}+(\rho+p) \dot{V}=\frac{1}{2}(\rho+p) \dot{V}=\frac{n(n-1)}{16 \pi G} \Omega_{n} H^{-n-1} \dot{H}^{2} \geq 0 .
$$

In the derivation of the above equation we have used the second Friedmann equation $\dot{H}=$ $-\frac{8 \pi G}{(n-1)}(\rho+p)$ and $\dot{V}=\partial_{t}\left(\Omega_{n} \tilde{r}_{A}^{n}\right)=\partial_{t}\left(\Omega_{n} H^{-n}\right)=-n \Omega_{n} H^{-n-1} \dot{H}$. In [21], the condition (28) without the term $W \dot{V}$ was obtained, which corresponds to the approximation $T=\frac{1}{2 \pi \tilde{r}_{A}}$. 
Now we consider a Schwarzschild black hole inside the apparent horizon in $(3+1)$ dimensional spacetime, whose mass is assumed to be small enough $M \ll \rho V$ so that the FRW metric remains unchanged (thereby $b=1$ ). Using the first Friedmann equation (6) in $(3+1)$-dimensional spacetime, this condition reduces to

$$
M H \ll \frac{\tilde{r}_{A}^{3} H^{3}}{2}=\frac{1}{2}
$$

where we set $G=1$. In a fluid with the energy density $\rho$ and the pressure $p$, the change rate of the black hole mass was obtained in [13]

$$
\dot{M}=4 \alpha r_{h}^{2}(\rho+p) M^{2}=-4 \alpha M^{2} \dot{H},
$$

where $r_{h}$ is the radius of the black hole horizon and $\alpha \sim O(1)$ is a positive numerical constant. The entropy of the black hole is $S_{b l}=4 \pi M^{2}$ [31], therefore

$$
\dot{S}_{b l}=-32 \pi \alpha M^{2} \dot{H}
$$

To protect the GSL, we require that the sum of the apparent horizon entropy, the ordinary matter entropy and the black hole entropy cannot decrease with time:

$$
\dot{S}_{h}+\dot{S}_{\rho}+\dot{S}_{b l} \geq 0
$$

Employing Eqs. (23), (24), (30), together with the Friedmann equation (6) and $b=1$, the condition to protect the GSL reads

$$
2 \pi \dot{H}\left(-16 \alpha M^{3}+\frac{\dot{H}}{2 H^{5}} \frac{1}{1-\epsilon}\right) \geq 0 .
$$

If the fluid surrounding the black hole is quintessence $\dot{H}<0$, the GSL holds always. If the fluid is the phantom type, $\dot{H}>0$, the GSL can also hold, because

$$
\frac{\dot{H}}{H^{2}} \frac{1}{1-\epsilon} \geq 32 \alpha M^{3} H^{3} \sim 0
$$

where the condition (29) and the positive temperature has been considered.

In the following, we are going to extend the discussion on the condition (27) to protect the GSL to generalized gravity theories. 


\section{A. Lovelock gravity}

The Lagrangian of the Lovelock gravity consists of the dimensionally extended Euler densities [32]

$$
L=\sum_{i=1}^{[n / 2]} c_{i} L_{i},
$$

where $c_{i}$ is an arbitrary positive constant and $L_{i}$ is the Euler density of a (2i)-dimensional manifold

$$
L_{i}=2^{-i} \delta_{\alpha_{1} \beta_{1} \cdots \alpha_{i i} \beta_{i}}^{\mu_{1} \nu_{1} \cdots \mu_{i} \nu_{i}} R_{\mu_{1} \nu_{1} \cdots \mu_{i} \nu_{i}}^{\alpha_{1} \beta_{1} \cdots \alpha_{i} \beta_{i}} .
$$

$L_{1}$ is just the Einstein-Hilbert term, and $L_{2}$ corresponds to the so called Gauss-Bonnet term. Using the FRW metric, we obtain Friedmann equations in $(n+1)$-dimensional spacetime

$$
\sum_{i=1}^{[n / 2]} \hat{c}_{i}\left(H^{2}\right)^{i}=\frac{16 \pi G}{n(n-1)} \rho
$$

and

$$
\sum_{i=1}^{[n / 2]} \hat{c}_{i} i\left(H^{2}\right)^{i-1}(\dot{H})=-\frac{8 \pi G}{(n-1)}(\rho+p)
$$

where

$$
\hat{c}_{i}=\frac{(n-2) !}{(n-2 i) !} c_{i}
$$

Since only one dynamic field $\rho$ in the first Friedmann equation (31), the first law on the horizon is described by the equilibrium thermodynamics $d_{p} S=0$. Two Friedmann equations (31) (32) make the energy flow $q=0$, so the horizon and energy are in thermodynamical equilibrium $b=1$. From two Friedmann equations (31) (32), one can find that the condition (27) always holds

$$
\frac{1}{2}(\rho+p) \dot{V}=\frac{n(n-1)}{16 \pi G} \Omega_{n} H^{-1-n} \sum_{i=1}^{[n / 2]} \hat{c}_{i} i\left(H^{2}\right)^{i-1} \dot{H}^{2} \geq 0 .
$$

The condition to protect the GSL in the Einstein gravity (28) can be recovered when $\hat{c}_{1}=1$, $\hat{c}_{i}=0(i>1)$. 


\section{B. Randall-Sundrum braneworld gravity}

We consider a $n$-dimensional brane embedded in a $(n+2)$-dimensional spacetime. Using the junction condition on the brane, we can obtain Friedmann equations

$$
\begin{gathered}
H^{2}=\frac{1}{4 n^{2}} \rho^{2}+\frac{1}{2 n^{2}} \lambda \rho \\
\dot{H}=-\frac{1}{4 n}(\rho+p)(\rho+\lambda)
\end{gathered}
$$

where the Randall-Sundrum fine-turning condition

$$
\frac{1}{4 n^{2}} \lambda^{2}+\frac{2 \Lambda_{n+2}}{n(n+1)}=0
$$

has been used. Using the junction condition into the (05) component of the field equation we can obtain the conserved equation

$$
\dot{\rho}+n H(\rho+p)=q=0
$$

which results that the horizon and energy inside the horizon are in thermodynamical equilibrium $b=1$.

We see that $\rho$ is the only freedom in the first Friedmann equation (34), so we only need to consider the equilibrium thermodynamics about the horizon. From (34) and (35), one can find that the condition (27) to protect the GSL always holds

$$
\frac{1}{2}(\rho+p) \dot{V}=\frac{2 n^{2} \Omega_{n}}{\sqrt{\lambda^{2}+4 n^{2} H^{2}}} H^{-n-1} \dot{H}^{2} \geq 0,
$$

The condition (37) can be reduced to the condition in Einstein gravity (28) at low energy $\rho \ll \lambda$.

\section{Nonlinear gravity}

For the nonlinear gravity $f(R)$, the Lagrangian is

$$
L=\frac{1}{16 \pi G} f(R)
$$

The variational principle gives equations of motion. Using the FRW metric, one can obtain Friedmann equations in $(n+1)$-dimensional space-time

$$
H^{2}=\frac{16 \pi G}{n(n-1)} \frac{1}{f^{\prime}}\left(\rho+\rho_{c} f^{\prime}\right)
$$




$$
\dot{H}=-\frac{8 \pi G}{(n-1)} \frac{1}{f^{\prime}}\left(\rho+\rho_{c} f^{\prime}+p+p_{c} f^{\prime}\right)
$$

where

$$
\begin{gathered}
\rho_{c}=\frac{1}{8 \pi G f^{\prime}}\left[-\frac{f-R f^{\prime}}{2}-n H f^{\prime \prime} \dot{R}\right] \\
p_{c}=\frac{1}{8 \pi G f^{\prime}}\left[\left(f-R f^{\prime}\right)-f^{\prime \prime} \ddot{R}+f^{\prime \prime \prime} \dot{R}^{2}+n(n-1) f^{\prime \prime} \dot{R}\right] .
\end{gathered}
$$

The prime denotes the derivate respect to $R$. Since $\frac{\partial H^{2}}{\partial \rho}$ is determined by dynamic field $f^{\prime}$ while not the horizon radius uniquely, one should consider the horizon described by non-equilibrium thermodynamics. One can select $\rho_{p}$ arbitrarily. For example, we select $\rho_{p}=\left(f^{\prime}, \rho_{c}\right)$. There is not the real matter field besides the ordinary matter $\rho$. It is important to observe that from the Friedmann equations, $\rho_{e} \equiv \rho_{c} f^{\prime}\left(p_{e} \equiv p_{c} f^{\prime}\right)$ acts as the density (pressure) of an effective energy component in $f(R)$ gravity.

The condition (27) reads

$$
(1-b) \dot{\rho}_{t} V+\left(1-\frac{b}{2}\right)\left(\rho_{t}+p_{t}\right) \dot{V} \geq 0
$$

where the total density (pressure) is $\rho_{t} \equiv \rho+\rho_{e}\left(p_{t} \equiv p+p_{e}\right)$. Solving $\rho_{t}$ and $p_{t}$ from two Friedmann equations (38) and (39), and substituting them into the above inequality, we find

$$
\frac{n(n-1) \Omega_{n}}{16 \pi G} H^{-(n+1)}\left[(1-b) H^{3} \dot{f}^{\prime}+2(1-b) f^{\prime} H^{2} \dot{H}+(2-b) f^{\prime} \dot{H}^{2}\right] \geq 0 .
$$

This inequality is the condition to protect the GSL in the $f(R)$ gravity. Now we like to point out a nontrivial observation that this condition may hold always. From two Friedmann equations (38) and (39), we find that $\dot{f}^{\prime}$ is related to the total energy flow $q_{t}$

$$
q_{t}=\frac{n(n-1)}{16 \pi G} H^{2} \dot{f}^{\prime}
$$

Since $G / f^{\prime}$ takes role as the effective Newton gravitational constant, the relation (41) means the energy fluid is determined by the evolvement of effective Newton gravitational constant. Consider the temperature of total energy $b T$, which in general can not equal to the temperature of horizon $T$. However, it is known that the experimental bounds acquires the Newton constant should be approximate constant [37]. From the relation (41), one can find that the big energy fluid is prohibited to protect the effective Newton constant as approximate constant. Thereby the thermodynamic fluid is small and the temperature of the horizon is very closed with the one of the energy source therein $b \sim 1$. This is more reasonable if two 
systems undergo some length of time. So the former two terms in the bracket of condition (40) may be neglected. Moreover, since the gravity is always attractive, we can impose $f^{\prime}>0$. Thus the last term in the bracket is positive and the GSL always holds. We also note that when the effective Newton constant is constant indeed $\dot{f}^{\prime}=0$ which leads $q_{t}=0$ then $b=1$, the GSL always holds, recovering the results of Einstein gravity.

It is interesting to show the entropy production $d_{i} S$ clearly. Recalling the conserved equation (10)

$$
\dot{\rho}_{e f f}=-n H\left(\rho_{e f f}+p_{e f f}\right)
$$

and using the first Friedmann equation (38), one can find that the l.h.s in Eq. (42) reads

$$
\begin{aligned}
\dot{\rho}_{e f f} & =\frac{n(n-1)}{16 \pi G}\left(\frac{\partial H^{2}}{\partial \rho} \dot{\rho}+\frac{\partial H^{2}}{\partial \rho_{p}} \dot{\rho}_{p}\right) \\
& =\frac{n(n-1)}{16 \pi G}\left(\frac{16 \pi G}{n(n-1)} \frac{1}{f^{\prime}} \dot{\rho}+\frac{\partial H^{2}}{\partial \rho_{p}} \dot{\rho}_{p}\right) \\
& =\frac{1}{f^{\prime}} \dot{\rho}+\frac{n(n-1)}{16 \pi G} \frac{\partial H^{2}}{\partial \rho_{p}} \dot{\rho}_{p}
\end{aligned}
$$

while by using the second Friedmann equation (39) the r.h.s in Eq. (42) reads

$$
-n H\left(\rho_{e f f}+p_{e f f}\right)=-n H\left[\frac{1}{f^{\prime}}\left(\rho_{t}+p_{t}\right)\right] \text {. }
$$

Employing the continuous equation (11) to Eqs. (43) and (44), one can find

$$
\frac{\partial H^{2}}{\partial \rho_{p}} \dot{\rho}_{p}=-\frac{16 \pi G}{n(n-1)} \frac{1}{f^{\prime}}\left[n H\left(\rho_{t}+p_{t}-\rho-p\right)+q\right] .
$$

Using the continuous equation (12) and substituting Eq. (45) into Eq. (20), we have

$$
\begin{aligned}
\delta Q & =n V H(\rho+p) d t-V q d t-V d t \frac{1}{\frac{\partial H^{2}}{\partial \rho}} \frac{\partial H^{2}}{\partial \rho_{p}} \dot{\rho}_{p}-V \frac{1}{\frac{\partial H^{2}}{\partial \rho}} \frac{\dot{H}^{2}}{H}+T \frac{16 \pi G}{n(n-1)} S_{E} d \frac{1}{\frac{\partial H^{2}}{\partial \rho}} \\
& =n V H\left(\rho_{t}+p_{t}\right) d t-V d t \frac{1}{\frac{\partial H^{2}}{\partial \rho}} \frac{\dot{H}^{2}}{H}+T S_{E} d f^{\prime} \\
& =n V H\left(\rho_{t}+p_{t}\right) d t-\frac{1}{2}\left(\rho_{t}+p_{t}\right) \dot{V}+T S_{E} d f^{\prime} \\
& =-d E+W_{t} d V+V q_{t} d t+T S_{E} d f^{\prime} .
\end{aligned}
$$

One can find clearly that the energy fluid $V q_{t} d t$ accounts for some part of the thermodynamical fluid. From Eqs. (21) and (46), the entropy production can be obtained

$$
\begin{aligned}
d_{i} S & =-\frac{1}{T} V q_{t} d t-S_{E} d f^{\prime} \\
& =\frac{-n \Omega_{n} H^{-n+1} d f^{\prime}\left[(n+1) H^{2}+\dot{H}\right]}{4 G\left(2 H^{2}+\dot{H}\right)} .
\end{aligned}
$$


The second equation has used the two Friedmann equations and Eq. (41). One can find $d_{i} S$ is not an exact form as desired and it is vanishing when the effective Newton gravitational constant $f^{\prime}$ is constant indeed. This is a reasonable result since the Einstein gravity is recovered in this situation. It should be noticed that this entropy production is different with the one given in Ref. [7, 10], where they have not considered the energy flux of effective energy $\rho_{e}$. If we really omit the energy flux of effective energy, the condition to protect GSL (26) is (for simplicity, we consider the $(3+1)$-dimensional space-time)

$$
\begin{aligned}
& \dot{S}_{h}+d_{i} \dot{S}+\dot{S}_{t}=-b\left[\partial_{t}(\rho V)+W \dot{V}\right]+V \dot{\rho}_{t}+\left(\rho_{t}+p_{t}\right) \dot{V} \\
= & \frac{\Omega_{3}}{32 \pi G H^{4}}\left\{\begin{array}{c}
12(1+b) H^{3} \dot{f}^{\prime}-3 b f \dot{H}+6(4+b) f^{\prime} \dot{H}^{2} \\
+12 H^{2}\left[(2+5 b) f^{\prime} \dot{H}-b \ddot{f}^{\prime}\right]-2 b H\left(\dot{f}+9 \dot{f}^{\prime} \dot{H}-6 f^{\prime} \ddot{H}\right)
\end{array}\right\} \geq 0,
\end{aligned}
$$

Obviously, the GSL can not be held always in this situation, which is a strong point to favor the present entropy production (21).

\section{Scalar-tensor gravity}

The general scalar-tensor theory of gravity is described by the Lagrangian

$$
L=F(\phi) R-\frac{1}{2} g^{\mu \nu} \partial_{\mu} \phi \partial_{\nu} \phi-V(\phi)
$$

where $F(\phi)$ is a positive continuous function of the scalar field $\phi$ and $V(\phi)$ is its potential. Using the FRW metric, we obtain Friedmann equations in $(n+1)$-dimensional space-time

$$
\begin{gathered}
H^{2}=\frac{16 \pi G}{n(n-1)} \frac{1}{F}\left(\rho+\rho_{f}+\rho_{c} F\right) \\
\dot{H}=\frac{8 \pi G}{(n-1)} \frac{1}{F}\left(\rho+p+\rho_{f}+p_{f}+\rho_{c} F+p_{c} F\right) .
\end{gathered}
$$

where the density and pressure of scalar field $\phi$ are

$$
\begin{aligned}
& \rho_{f}=\frac{1}{2} \dot{\phi}^{2}+V(\phi) \\
& p_{f}=\frac{1}{2} \dot{\phi}^{2}-V(\phi),
\end{aligned}
$$

and $\rho_{e} \equiv \rho_{c} F\left(p_{e} \equiv p_{c} F\right)$ can be understood as effective density (pressure) of the energy component in scalar-tensor theory:

$$
\rho_{c}=-\frac{n}{8 \pi G F} H \dot{F}
$$




$$
p_{c}=\frac{1}{8 \pi G F}[\ddot{F}+(n-1) H \dot{F}] .
$$

Obviously, we need to consider the non-equilibrium thermodynamics of horizon. We select $\rho_{p}=\left(\rho_{f}, F, \rho_{e}\right)$.

Consider the condition (27) for GSL

$$
(1-b) \dot{\rho}_{t} V+\left(1-\frac{b}{2}\right)\left(\rho_{t}+p_{t}\right) \dot{V} \geq 0
$$

where the total density (pressure) is $\rho_{t} \equiv \rho+\rho_{f}+\rho_{e}\left(p_{t} \equiv p+p_{f}+p_{e}\right)$. Solving $\rho_{t}$ and $p_{t}$ from two Friedmann equations (47) and (48), and substituting them into the above inequality, we find that the inequality can be reduced to,

$$
\frac{n(n-1) \Omega_{n}}{16 \pi G} H^{-(n+1)}\left[(1-b) H^{3} \dot{F}+2(1-b) F H^{2} \dot{H}+(2-b) F \dot{H}^{2}\right] \geq 0
$$

The effective Newton gravitational constant in the scalar tensor theory is taken as $G / F$. From two Friedmann equations (47) and (48), the dynamical Newton constant is related to the energy flow

$$
q_{t}=\frac{n(n-1)}{16 \pi G} H^{2} \dot{F}
$$

The condition (49) and the relation (50) are the same as those in the $f(R)$ gravity. As observed in the $f(R)$ gravity, we can think that the GSL for scalar-tensor theory always holds, since we can impose $F>0$ which means the gravity is always attractive, and the big energy fluid is prohibited to protect the effective Newton constant as approximate constant under experimental bounds, thereby the temperature of the horizon is very closed with the one of the energy source therein $b \sim 1$. Moreover, it should be noticed that, actually $f(R)$ gravity is a special scalar-tensor theory by introducing the scalar field $\phi=R$ and potential $V=\phi f^{\prime}-f$ and choosing the Brans-Dick parameter $\omega=0$ ([33, 34] - see [35] for a review).

Now we will evaluate the entropy production. The process is similar to the one of nonlinear gravity. Recalling the continuous equation (10) and using the first Friedmann equation (47), one can find that the l.h.s in Eq. (42) reads

$$
\dot{\rho}_{e f f}=\frac{1}{F} \dot{\rho}+\frac{n(n-1)}{16 \pi G} \frac{\partial H^{2}}{\partial \rho_{p}} \dot{\rho}_{p} .
$$

Using the second Friedmann equation (48) the r.h.s in Eq. (42) reads

$$
-n H\left(\rho_{e f f}+p_{e f f}\right)=-n H \frac{1}{F}\left(\rho_{t}+p_{t}\right)
$$


Employing the continuous equation (11) to Eqs. (51) and (52), one can find

$$
\frac{\partial H^{2}}{\partial \rho_{p}} \dot{\rho}_{p}=-\frac{16 \pi G}{n(n-1)} \frac{1}{F}\left[n H\left(\rho_{t}+p_{t}-\rho-p\right)+q\right] .
$$

By substituting Eq. (53) into Eq. (20), one can obtain

$$
\delta Q=-d E+W_{t} d V+V q_{t} d t+T S_{E} d F .
$$

Thus the entropy production can be obtained from Eqs. (21) and (50)

$$
d_{i} S=\frac{-n \Omega_{n} H^{-n+1} d F\left[(n+1) H^{2}+\dot{H}\right]}{4 G\left(2 H^{2}+\dot{H}\right)} .
$$

One can find $d_{i} S$ is not an exact form and it is vanishing when the effective Newton gravitational constant $F$ is constant indeed.

\section{SUMMARY}

In this paper, we have examined the GSL in generalized theories of gravity. We have adopted the procedure developed in [12] to obtain the entropy on the horizon. In studying the GSL, we have examined the evolution of the entropy contributed by all matter, field and energy contents. We have derived a universal condition to protect the GSL and examined its validity. In Einstein gravity, (even in the phantom-dominated universe with a Schwarzschild black hole), Lovelock gravity, and braneworld gravity, we show that the condition to keep the GSL can always be satisfied. In $f(R)$ gravity and scalar-tensor gravity, the condition to protect the GSL can also hold under the consideration that the gravity is always attractive and the energy fluid between the horizon and total energy source therein is very small to protect the effective Newton constant as the approximate constant satisfying the experimental bounds. The same requirement for the $f(R)$ gravity to hold the GSL as that of the scalar-tensor gravity shows again that $f(R)$ gravity is a special scalar-tensor theory.

\section{Acknowledgment}

This work was partially supported by the NSFC, Shanghai Education Commission, Science and Technology Commission. This work was also supported by the NSFC under Grant Nos. 10575068 and 10604024, the Shanghai Research Foundation No. 07dz22020, the CAS 
Knowledge Innovation Project Nos. KJcx.syw.N2, the Shanghai Education Development Foundation, and the Innovation Foundation of Shanghai University.

[1] T. Jacobson, Phys. Rev. Lett. 75 (1995) 1260.

[2] T. Padmanabhan, Class. Quant. Grav. 19 (2002) 5387; Phys. Rep. 406 (2005) 49.

[3] A. V. Frolov, L. Kofman, JCAP 0305 (2003) 009.

[4] U. H. Danielsson, Phys. Rev. D 71 (2005) 023516.

[5] R. Bousso, Phys. Rev. D 71 (2005) 064024.

[6] M. Akbar and R. G. Cai, Phys. Rev. D 75 (2007) 084003.

[7] R. G. Cai, L. M. Cao, Phys. Rev. D 75 (2007) 064008.

[8] R. G. Cai, L. M. Cao, Nucl. Phys. B 785 (2007) 135.

[9] A. Sheykhi, B. Wang, and R. G. Cai, Nucl. Phys. B 779 (2007) 1; A. Sheykhi, B. Wang, and R. G. Cai, Phys. Rev. D 76 (2007) 023515; S. F. Wu, G. H Yang, and P. M. Zhang, arXiv:0710.5394.

[10] C. Eling, R. Guedens, T. Jacobson, Phys. Rev. Lett. 96 (2006) 121301.

[11] M. Akbar and R. G. Cai, Phys. Lett. B 635 (2006) 7; Phys. Lett. B 648 (2007) 243.

[12] S. F. Wu, B. Wang, and G. H Yang, Nucl. Phys. B in press, arXiv:0711.1209.

[13] E. Babichev, V. Dokuchaev, Yu. Eroshenko, Phys. Rev. Lett. 93 (2004) 021102; J. Exp. Theor. Phys. 100 (2005) 528.

[14] M. R. Setare and S. Shafei, JCAP 09 (2006) 011; M.R. Setare, Phys. Lett. B 641 (2006) 130;

W. Buchmüller and J. Jaeckel, astro-ph/0610835.

[15] M. D. Pollock, T. P. Singh, Class. Quantum Grav. 6 (1989) 901.

[16] P. C. W. Davies, Class. Quant. Grav 4 (1987) L225.

[17] H. Mohseni Sadjadi, Phys. Rev. D 73 (2006) 063525.

[18] G. Izquierdo, D. Pavón, Phys. Lett. B 639 (2006) 1.

[19] H. Mohseni Sadjadi, Phys. Lett. B 645 (2007) 108.

[20] B. Wang, Y. Gong, Elcio Abdalla, Phys. Rev. D 74 (2006) 083520.

[21] J. Zhou, B. Wang, Y. Gong, and E. Abdalla, Phys. Lett. B 652 (2007) 86.

[22] H. Mohseni Sadjadi, Phys. Rev. D 76 (2007) 104024.

[23] R. G. Cai and S. P. Kim, JHEP 0502 (2005) 050. 
[24] R. G. Cai, Phys. Lett. B 582 (2004) 237.

[25] R. M. Wald, Phys. Rev. D 48 (1993) R3427; G. Cognola, E. Elizalde, S. Nojiri, S. D. Odintsov, S. Zerrbini, JCAP 0502 (2005) 010; I. Brevik, S. Nojiri, S. D. Odintsov, and L. Vanzo, Phys. Rev. D 70 (2004) 043520.

[26] R. G. Cai and Y. S. Myung, Phys. Rev. D 56 (1997) 3466.

[27] Y. Gong and A. Wang, Phys. Rev. Lett. 99 (2007) 211301.

[28] C. Eling, arXiv:0806.3165.

[29] E. Elicalde, and P. J. Silva, arXiv:0804.3721.

[30] G. Izquierdo, D. Pavon, Phys. Lett. B 633 (2006) 420.

[31] J. D. Bekenstein, Phys. Rev. D 12 (1975) 3077.

[32] D. Lovelock, J. Math. Phys. 12 (1971) 498.

[33] C. H. Brans and R. H. Dicke, Phys. Rev. 124 (1961) 925.

[34] P. G. Bergmann, Int. J. Theor. Phys. 1 (1968) 25; R. V. Wagoner, Phys. Rev. D 1 (1970) 3209; K. Nordvedt, Astrophys. J. 161 (1970) 1059.

[35] V. Faraoni, Cosmology in Scalar-Tensor Gravity (Kluwer Academic, Dordrecht, 2004).

[36] S. A. Hayward, Class. Quant. Grav. 15 (1998) 3147; S. A. Hayward, S. Mukohyama, and M. C. Ashworth, Phys. Lett. A 256 (1999) 347.

[37] J. P. Ozan, Rev. Mod. Phys. 75 (2003) 403. 